\title{
Pion pole and transversity effects in hard exclusive meson leptoproduction
}

\section{Sergey Goloskokov*}

Bogoliubov Laboratory of Theoretical Physics, Joint Institute for Nuclear Research, Dubna 141980, Moscow region, Russia

E-mail: goloskkvetheor.jinr.ru

We investigate exclusive electroproduction of vector and pseudoscalar mesons at large photon virtuality $Q^{2}$. These reactions were analyzed within the handbag approach where amplitudes factorize into hard subprocesses and generalized parton distributions (GPDs).

The essential role of transversity effects were found in pseudoscalar and light vector meson leptoproduction. These contributions are important at low $Q^{2}$ and determined by twist- 3 effects accompanied by transversity GPDs. The transversity contributions lead to large transverse cross sections for most reactions of pseudoscalar meson leptoproduction. The transversity effects in vector meson production are visible in spin observables.

We consider spin effects in the $\omega$ and $\rho^{0}$ leptoproduction reactions. It is shown that the pion pole contribution is very important in the $\omega$ production. Such effects in the $\rho^{0}$ channel are much smaller. Our results on spin asymmetries and spin density matrix elements in these reactions were found to be in good agreement with HERMES data.

XXII International Baldin Seminar on High Energy Physics Problems,

15-20 September 2014

JINR, Dubna, Russia

\footnotetext{
${ }^{*}$ Speaker.
} 


\section{1. introduction}

Our investigations of hard electroproduction of vector mesons [1] were based on the handbag approach where the amplitude of meson production at high $Q^{2}$ factorizes into hard meson electroproduction off partons, and GPDs [2]. The hard subprocesses were analysed within the modified perturbative approach [3] where we considered the quark transverse degrees of freedom accompanied by the Sudakov suppressions. This approach describes successfully the data on $\rho^{0}$ and $\phi$ electroproduction and discussed in section 2.

The amplitudes of the pseudoscalar meson (PM) leptoproduction in the leading twist approximation are sensitive to GPDs $\widetilde{H}$ and $\widetilde{E}$. It was found that these contributions were not sufficient to describe spin effects in the PM production at sufficiently low $Q^{2}$ [4]. To be consistent with experiment, essential contributions from the transversity GPDs $H_{T}, \bar{E}_{T}$ are needed [5]. Within the handbag approach the transversity GPDs go together with the twist- 3 meson wave function. We discuss in section 3 the role of transversity effects in the PM leptoproduction at HERMES and CLAS energies. We show that the transversity GPDs lead to a large transverse cross section for most reactions of the PM leptoproduction [5], which exceed the leading twist longitudinal cross section. The role of transversity effects were analysed in the vector meson (VM) leptoproduction too. The transversity GPDs were found to be important in the spin density matrix elements (SDMEs) and spin asymmetries of the VM leptoproduction with a transversely polarized target. The obtained results are in good agreement with CLAS, HERMES and COMPASS data.

The HERMES data on SDMEs for the $\omega$ production indicated strong contributions from unnatural parity exchanges. Using GPDs from our analyses of the hard meson leptoproduction we investigated $\omega$ SDMEs [6]. It was found that the pion pole (PP) contribution plays an important role in the $\omega$ production and is essential in explanation of the large unnatural-parity effects observed by HERMES. Based on our approach we found $\omega$ SDMEs to be in good agreement with the HERMES experimental results [7]. The PP contribution to the $\rho^{0}$ production is much smaller with respect to the $\omega$ case. We discuss the PP effects in section 4.

\section{Handbag approach. Vector meson leptoproduction}

Within the handbag approach the meson production amplitude at sufficiently high photon virtuality $Q^{2}$ is factorized [2] into a hard subprocess amplitude $\mathscr{H}$ and GPDs $F$ which contain information on the hadron structure. In the forward limit and zero skewness GPDs are equivalent to ordinary Parton Distribution Functions (PDFs). With the help of sum rules they are connected with hadron form factors, and information on the parton angular momenta can be extracted.

The leading contributions to the meson production amplitude off non-flip proton can be described in terms of various parton effects

$$
M_{\mu^{\prime}+, \mu+} \propto \int_{-1}^{1} d x \mathscr{H}_{\mu^{\prime}+, \mu+}^{a} F^{a}(x, \xi, t) .
$$

Here $a$ is a flavor factor, $\mathscr{H}_{\mu^{\prime}+, \mu+}^{a}$ is a hard meson electroproduction amplitude off partons with the same helicities, $\mu$ and $\mu^{\prime}$ are helicities of the photon and produced meson. In the VM production we have $F^{a}$ GPDs contributions from gluons, quarks and sea. In the PM production polarized GPDs $\tilde{F}^{a}$ give contribution. 
The subprocess amplitude is calculated within the modified perturbative approach [3]. We consider the $k_{\perp}^{2} / Q^{2}$ corrections in the propagators of the hard subprocess amplitude $\mathscr{H}$ together with the nonperturbative $\mathbf{k}_{\perp}$-dependent meson wave function [8]. The power corrections can be regarded as an effective consideration of the higher twist effects. The gluonic corrections are treated in the form of the Sudakov factors whose resummation can be done in the impact parameter space [3].

The GPDs are estimated using the double distribution representation [9]

$$
F_{a}(\bar{x}, \xi, t)=\int_{-1}^{1} d \beta \int_{-1+|\beta|}^{1-|\beta|} d \alpha \delta(\beta+\xi \alpha-\bar{x}) f_{a}(\beta, \alpha, t) .
$$

which connects GPDs $F$ with PDFs $h$ through the double distribution function

$$
f_{a}(\beta, \alpha, t)=h_{a}(\beta, t) \frac{\Gamma\left(2 n_{i}+2\right)}{2^{2 n_{i}+1} \Gamma^{2}\left(n_{i}+1\right)} \frac{\left[(1-|\beta|)^{2}-\alpha^{2}\right]^{n_{i}}}{(1-|\beta|)^{2 n_{i}+1}} .
$$

Here $\mathrm{n}=1$ for valence quarks and $\mathrm{n}=2$ for gluon and sea contributions.

The $t$ - dependence in PDFs $h$ is considered in the Regge form

$$
h(\beta, t)=N e^{b_{0} t} \beta^{-\alpha(t)}(1-\beta)^{n},
$$

and $\alpha(t)$ is the corresponding Regge trajectory. The parameters in (2.4) are obtained from the known information about PDFs [10] e.g, or from the nucleon form factor analysis [11].
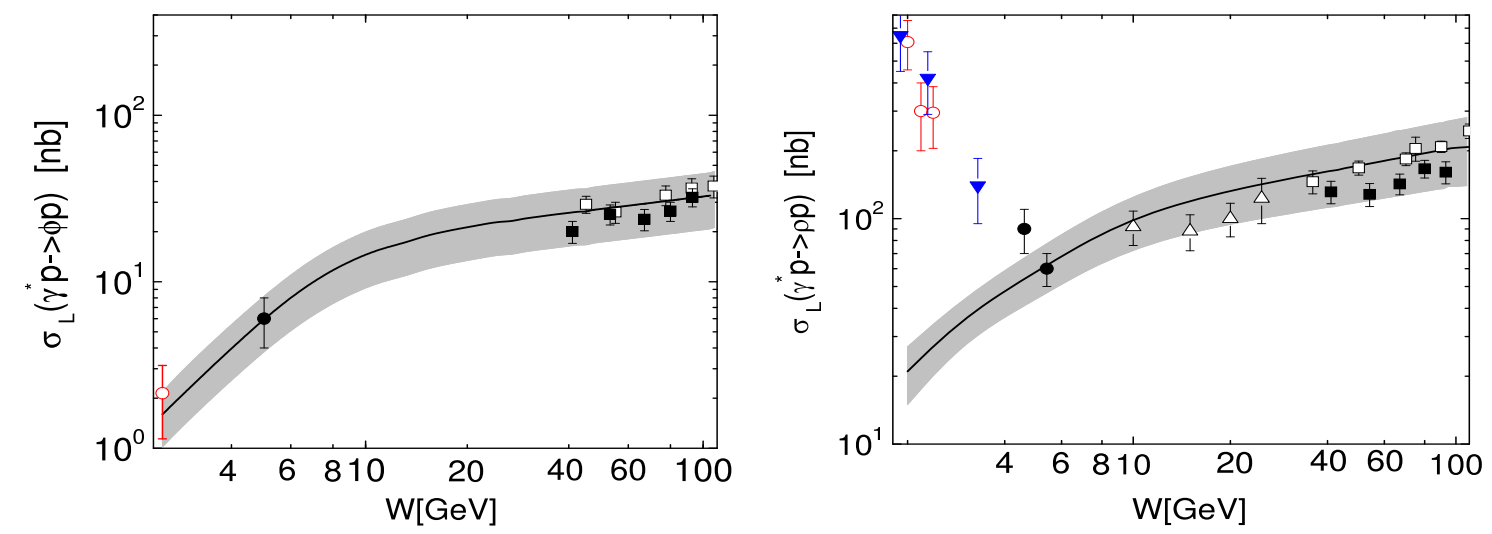

Figure 1: Left: The longitudinal cross section for $\phi$ at $Q^{2}=3.8 \mathrm{GeV}^{2}$. Data: HERMES (solid circle), ZEUS (open square), H1 (solid square), open circle- CLAS data point. Right: The longitudinal cross section for $\rho$ production at $Q^{2}=4.0 \mathrm{GeV}^{2}$. Data: HERMES (solid circle), ZEUS (open square), H1 (solid square), E665 (open triangle), CLAS- open circles, CORNEL -solid triangle

The handbag approach was successfully applied to light meson leptoproduction [1]. The cross sections and spin observables of light VM leptoproduction were found to be in good agreement with HERMES, COMPASS and HERA data. As an example, in Fig. 1, (left), we show our results for $W$ dependence of the $\phi$ leptoproduction at fixed $Q^{2}$ which reproduce the experimental data in the whole range from CLAS to HERA energies. This shows that gluon and sea GPDs work well from small to large $x$ - Bjorken. In Fig. 1, (right), we show a similar plot for the $\rho$ production. We see that the model describes the $\rho$ meson leptoproduction quite well for $W>4 \mathrm{GeV}$. The rapid growth of the cross section at lower energies has not been understood within the handbag model. 


\section{Transversity effects in light meson leptoproduction.}

Exclusive electroproduction of PM was studied within the handbag approach $[4,5]$. At the leading-twist accuracy the PM production is only sensitive to GPDs $\widetilde{H}$ and $\widetilde{E}$ which contribute to the amplitudes for longitudinally polarized virtual photons [4]. Such contributions are not sufficient to describe the experimental results on electroproduction of PM at low $Q^{2}$. We can show this from the $A_{U T}^{\sin \left(\phi_{s}\right)}$ asymmetry, Fig. 2.

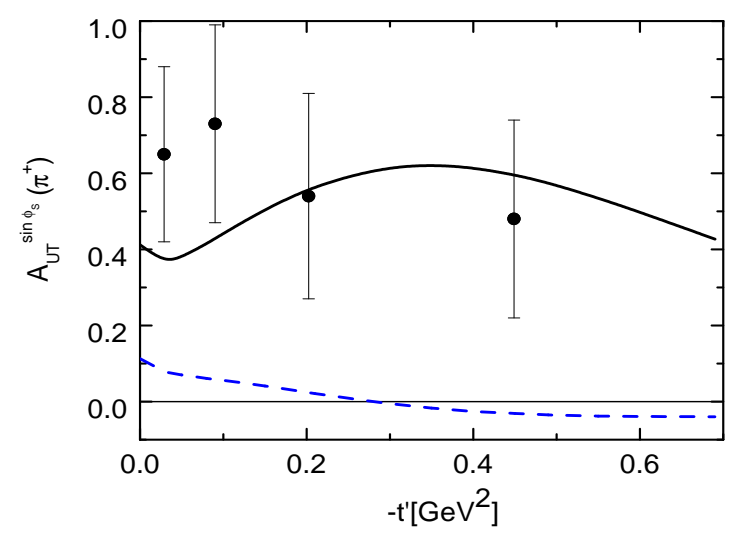

Figure 2: $A_{U T}^{\sin \left(\phi_{s}\right)}$ asymmetry: solid line prediction of our approach. Dashed line- without twist-3 effects.

This asymmetry is proportional to $M_{0-,++}$ and $M_{0+, 0+}$ interference

$$
A_{U T}^{\sin \left(\phi_{s}\right)} \propto \operatorname{Im}\left[M_{0-,++}^{*} M_{0+, 0+}\right] .
$$

In the handbag approach the amplitude $M_{0-,++} \propto t^{\prime}$. Small PP contribution to $M_{0-,++}$ can not explain this asymmetry- (dashed line in Fig. 2). Thus, the new sufficiently large contribution to the $M_{0-,++}$ amplitude is needed.

It was found that at low $Q^{2}$ the PM leptoproduction data also require contributions from transversity GPDs $H_{T}$ and $\bar{E}_{T}$ which determine the amplitudes $M_{0-,++}$ and $M_{0+,++}$, respectively. Within the handbag approach the transversity GPDs are accompanied by a twist-3 meson wave function in the hard amplitude $\mathscr{H}[5]$ which is the same for both the $M_{0 \pm,++}^{M, t w-3}$ amplitudes

$$
M_{0-,++}^{M, t w-3} \propto \int_{-1}^{1} d \bar{x} \mathscr{H}_{0-,++}(\bar{x}, \ldots) H_{T}^{M} ; M_{0+,++}^{M, t w-3} \propto \frac{\sqrt{-t^{\prime}}}{4 m} \int_{-1}^{1} d \bar{x} \mathscr{H}_{0-,++}(\bar{x}, \ldots) \bar{E}_{T}^{M} .
$$

The $H_{T}$ GPDs in the forward limit and $\xi=0$ are equal to transversity PDFs $\delta$. We parameterize the PDF $\delta$ by using the model [12]

$$
H_{T}^{a}(x, 0,0)=\delta^{a}(x) ; \quad \text { and } \quad \delta^{a}(x)=C N_{T}^{a} x^{1 / 2}(1-x)\left[q_{a}(x)+\Delta q_{a}(x)\right] .
$$

The information on $\bar{E}_{T}$ is obtained now only in the lattice QCD [13]. The lower moments of $\bar{E}_{T}^{u}$ and $\bar{E}_{T}^{d}$ were found to be quite large, have the same sign and a similar size. We parameterize $\bar{e}_{T}$ PDF in the form (2.4) with parameters determined from the lattice estimations. The double distribution is used to calculate GPDs as before. Note that $H_{T}^{u}$ and $H_{T}^{d}$ GPDs have different signs. 
These properties of GPDs provide an essential compensation of the $\bar{E}_{T}$ contribution in the $\pi^{+}$ amplitude, but $H_{T}$ effects are not small there. For the $\pi^{0}$ production we have the opposite case the $\bar{E}_{T}$ contributions are large and the $H_{T}$ effects are smaller.

We present here our results on the PM leptoproduction based on the handbag approach. In calculation, we use the leading contributions together with the transversity effects (3.2) which are essential at low $Q^{2}$. In Fig. 3 (left), we present the model results for the $\pi^{0}$ production cross section [5]. At small momentum transfer the $H_{T}$ contribution is visible and provides a nonzero cross section. At $-t^{\prime} \sim 0.2 \mathrm{GeV}^{2}$ the $\bar{E}_{T}$ contribution becomes essential and gives a maximum in the cross section. A similar contribution from $\bar{E}_{T}$ is observed in the interference cross section $\sigma_{T T}$. The fact that we describe well both unseparated $\sigma$ and $\sigma_{T T}$ cross sections can indicate that transversity effects were probably observed in CLAS [14]. In Fig. 3 (right), we show the $\eta$ and $\pi^{0}$ cross section ratio obtained in the model [5]. At small momentum transfer this ratio is controlled by the $H_{T}$ contribution. At larger $-t$ the $E_{T}$ contributions become important. The value about $1 / 3$ for the cross section ratio in the momentum transfer $-t^{\prime}>0.2 \mathrm{GeV}^{2}$ is a consequence of the flavor structure of the $\eta$ and $\pi^{0}$ amplitudes. This result was confirmed by the preliminary CLAS data [15].
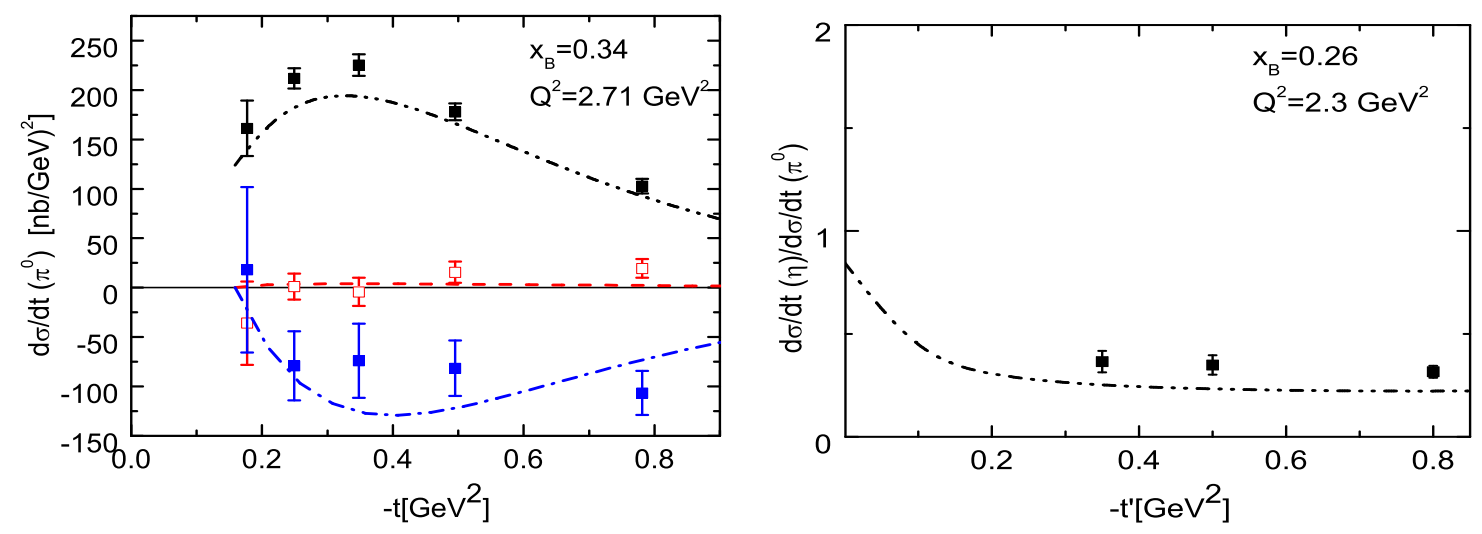

Figure 3: Left: $\pi^{0}$ production in the CLAS energy range together with the data [14]. Dashed-dot-dotted line- $\sigma=\sigma_{T}+\varepsilon \sigma_{L}$, dashed line- $\sigma_{L T}$, dashed-dotted- $\sigma_{T T}$. Right: $\eta / \pi^{0}$ production ratio in the CLAS energy range together with preliminary data [15].

Now we show some our results for transversity effects in the VM leptoproduction. In this case, the transversity $M_{0-,++}$ and $M_{0+,++}$ amplitudes have the form (3.2) but they are parametrically about 3 times smaller [16] with respect to the PM amplitudes. In calculations, we use the same parameterizations for transversity GPDs $H_{T}$ and $\bar{E}_{T}$ which were used in our study of the PM leptoproduction and can be found in $[5,16]$.

The importance of the transversity GPDs was examined in the SDMEs and asymmetries measured with a transversely polarized target. The $\bar{E}_{T}$ contribution is essential in some SDMEs. Really,

$$
r_{00}^{5} \sim \operatorname{Re}\left[M_{0+, 0+}^{*} M_{0+,++}\right] ; \quad r_{00}^{1} \sim-\left|M_{0+,++}\right|^{2} ; \quad M_{0+,++}=<\bar{E}_{T}>.
$$

Our results [16] for these SDMEs are shown in Fig.4, (left). They reproduce HERMES data [17] well. 

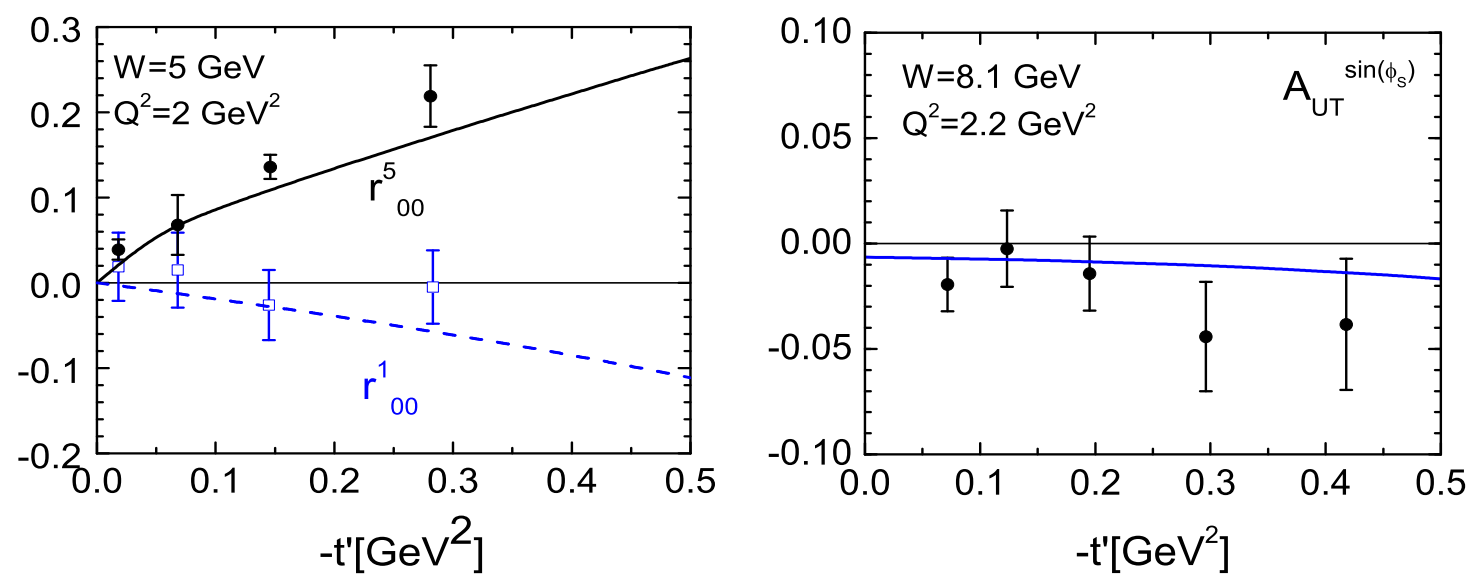

Figure 4: Left:Transversity effects at SDMEs at $W=5 \mathrm{GeV}$ together with HERMES data [17]. Right; Transversity effects in $A_{U T}^{\sin \left(\phi_{s}\right)}$ moment of asymmetry at COMPASS together with data [18]

The $\sin \left(\phi_{s}\right)$ moment of the $A_{U T}$ asymmetry is determined by the $H_{T}$ GPDs.

$$
A_{U T}^{\sin \left(\phi_{s}\right)} \sim \operatorname{Im}\left[M_{0-,++}^{*} M_{0+, 0+}\right] ; \quad M_{0-,++}=<H_{T}>
$$

This asymmetry is found to be not small at COMPASS [16] and compatible with the data [18], Fig. 4, (right). Thus, we see that the transversity effects are important in the description of PM and VM production at CLAS, HERMES and COMPASS energy. Our results are compatible with experimental data.

\section{Large unnatural parity effects in $\omega$ production. Pion pole effects.}

The HERMES data on the spin density matrix element for the $\omega$ production indicate strong contributions from unnatural parity contribution. The natural and unnatural parity amplitudes can be determined as

$$
\begin{aligned}
M_{\mu^{\prime} v^{\prime}, \mu v}^{N} & =\frac{1}{2}\left[M_{\mu^{\prime} v^{\prime}, \mu v}+(-1)^{\mu-\mu^{\prime}} M_{-\mu^{\prime} v^{\prime},-\mu v}\right] \\
M_{\mu^{\prime} v^{\prime}, \mu v}^{U} & =\frac{1}{2}\left[M_{\mu^{\prime} v^{\prime}, \mu v}-(-1)^{\mu-\mu^{\prime}} M_{-\mu^{\prime} v^{\prime},-\mu v}\right] .
\end{aligned}
$$

In most reactions the unnatural parity (UP) contributions are small with respect to the natural one. However, in the $\omega$ production at HERMES [7] it was found an unusual result: the ratio of the unnatural to the natural parity cross section

$$
U_{1}=2 \frac{d \sigma^{U}\left(\gamma_{T}^{*} \rightarrow V_{T}\right)+\varepsilon d \sigma^{U}\left(\gamma_{L}^{*} \rightarrow V_{L}\right)}{d \sigma},
$$

which was expected to be small, was found to be larger than unity, see Fig.5, (left).

Using GPDs from our analyses of hard meson leptoproduction we investigate [6] $\omega$ SDMEs measured by the HERMES Collaboration [7]. It was found that the PP contribution, Fig.5, (right), 

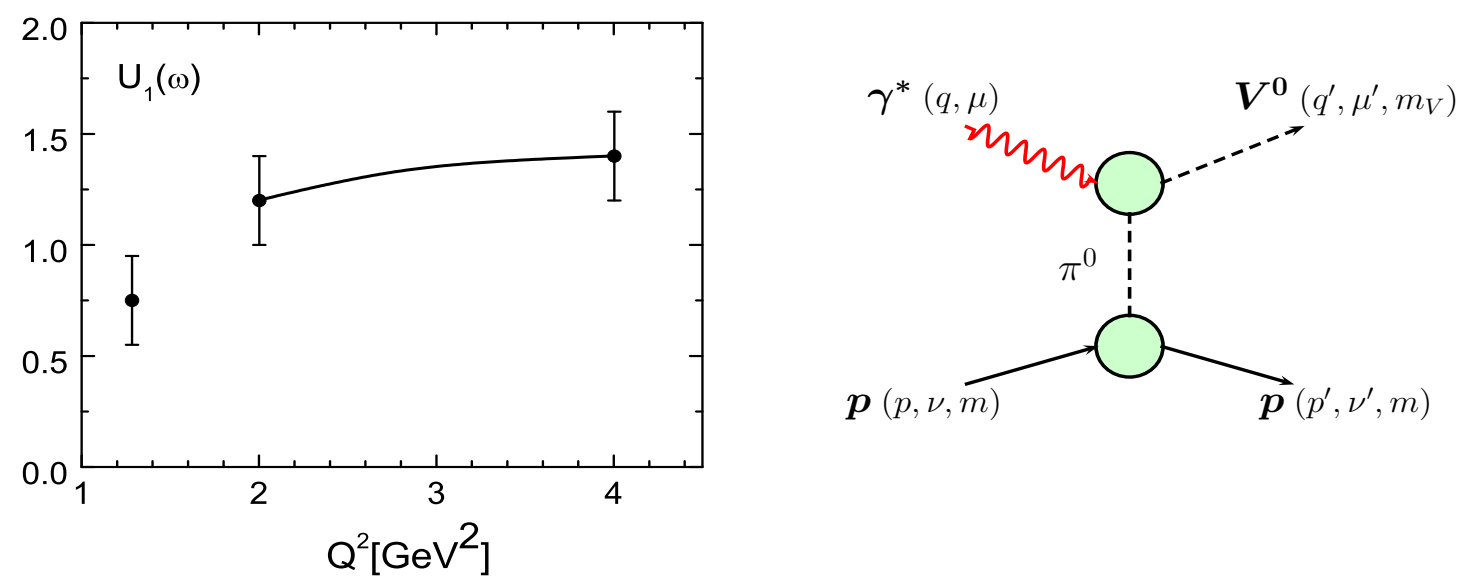

Figure 5: Left: $U_{1}$-the unnatural to natural parity cross section ratio at HERMES [7]. Right: the PP contribution in the $\omega$ production.

which has unnatural parity nature gives an important effect in the $\omega$ production. The PP contribution to helicity amplitudes is controlled by the $V \pi^{0} \gamma$ transition form factor. The PP contribution to helicity ampitudes looks like as follows:

$$
M_{++,++}^{\text {pole }} \sim \frac{\rho_{\pi V}}{t-m_{\pi}^{2}} \frac{m \xi Q^{2}}{\sqrt{1-\xi^{2}}} ; \quad M_{+-,++}^{\text {pole }} \sim-\frac{\rho_{\pi V}}{t-m_{\pi}^{2}} \frac{\sqrt{-t^{\prime}} Q^{2}}{2}
$$

with

$$
\rho_{\pi V} \sim g_{\pi V}\left(Q^{2}\right) g_{\pi N N} F_{\pi N N}(t) .
$$

The transition form factor $g_{\pi V}(0)$ is determined from the VM radiative decay

$$
\Gamma(V \rightarrow \pi \gamma) \sim \frac{\alpha_{e l m}}{24}\left|g_{\pi V}(0)\right|^{2} M_{V}^{3} .
$$

We find

$$
\left|g_{\pi \omega}(0)\right|=2.3 \mathrm{GeV}^{-1} ; \quad\left|g_{\pi \rho}(0)\right|=.85 \mathrm{GeV}^{-1} .
$$

This means that $\left|g_{\pi \omega}\right|$ is about 3 times larger with respect to $\left|g_{\pi \rho}\right|$ and we should observe large PP effects in $\omega$ and small in $\rho$ production. The $Q^{2}$ dependence of $g_{\pi V}\left(Q^{2}\right)$ was extracted from the $U_{1}$ data at $Q^{2}<4 \mathrm{GeV}^{2}$ [6], Fig.5 (left).

In what follows we will discuss a comparison of our results on PP effects in the $\omega$ and $\rho$ production with data at HERMES energy. The natural and unnatural parity asymmetry $P$

$$
P=\frac{d \sigma^{N}\left(\gamma_{T}^{*} \rightarrow V_{T}\right)-d \sigma^{U}\left(\gamma_{T}^{*} \rightarrow V_{T}\right)}{d \sigma^{N}\left(\gamma_{T}^{*} \rightarrow V_{T}\right)+d \sigma^{U}\left(\gamma_{T}^{*} \rightarrow V_{T}\right)}
$$

is an important example. If the UP contribution is small, we find $P \sim 1$. If it is large, we have rather a different value for the $P$ asymmetry. We find that with the PP contribution the $\omega$ asymmetry $P \sim-0.5$ (full line) in agreement with experiment. While neglecting the PP contribution we obtain $P \sim 0.5$ (dashed line). Our results together with the HERMES data for $\omega$ are shown in Fig.6, (left). In this figure we show for comparison the model results for CLAS energy $W=3.5 \mathrm{GeV}$ by the 
dotted line and for COMPASS energy $W=8 \mathrm{GeV}$ by the dash-dotted curve. It can be seen that at COMPASS energies PP effects are rather small for $\omega$. For the $\rho$ production PP effects are small and the asymmetry $P$ is close to unity -see Fig.6, (right).
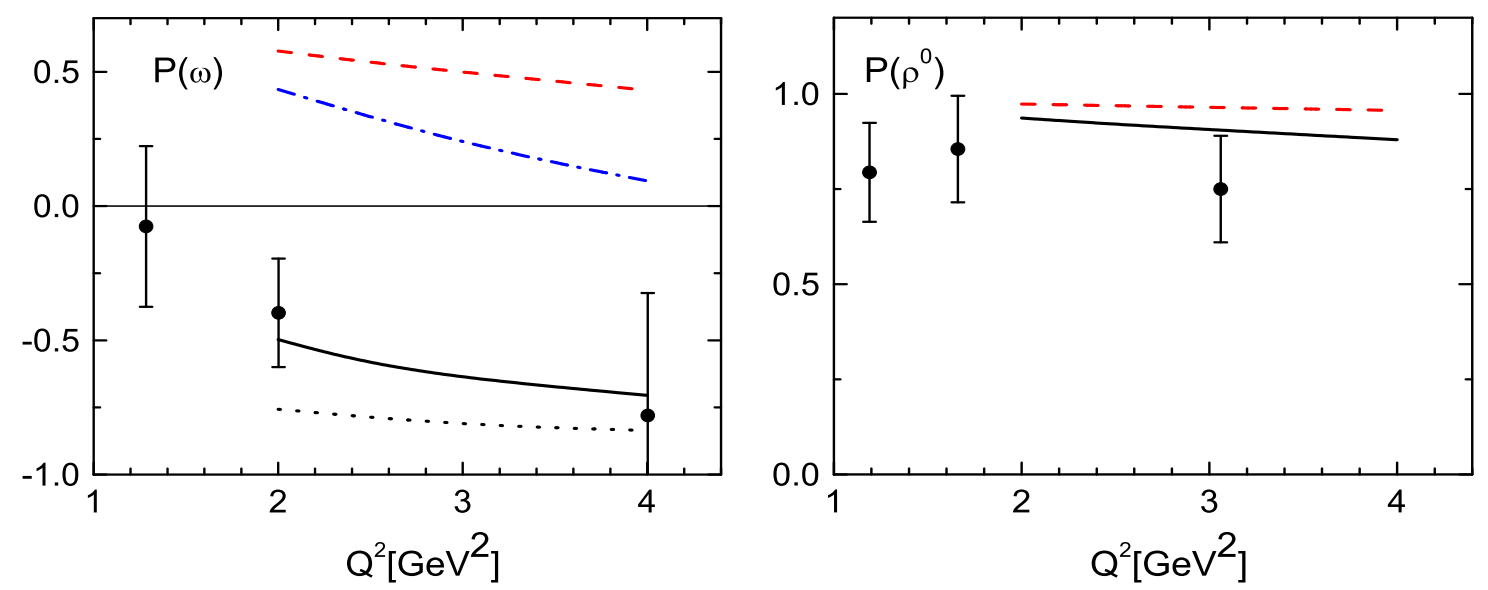

Figure 6: Left: $P(\omega)$ asymmetry at HERMES. Black solid line- with PP, Red-dashed line -without PP. Black dotted line-for $W=3.5 \mathrm{GeV}$ (CLAS), Blue dashed-dotted line for $W=8 \mathrm{GeV}$ (COMPASS). Right: $P\left(\rho^{0}\right)$ at HERMES. Black solid with PP, Red-dashed -without PP.

Interesting effects are observed in the ratio of the longitudinal and the transverse cross section

$$
R \simeq \frac{d \sigma\left(\gamma_{L}^{*} \rightarrow V_{L}\right)}{d \sigma\left(\gamma_{T}^{*} \rightarrow V_{T}\right)}
$$

for the $\omega$ and $\rho$ production. The PP give an essential contribution to amplitudes with transversely polarized protons (4.3). As a result, the PP effects lead to a quite small $R \sim 0.3$ ratio for the $\omega$ production at HERMES. This ratio is about $R \sim 1.2$ for the $\rho$ production and close to the $\omega$ case without the PP contribution, see Fig. 7, (left). In this figure, we show for comparison our results for the $R$ ratio for the $\omega$ production at CLAS and COMPASS energies.

The model results for SDME

$$
r_{00}^{04}=\frac{d \sigma\left(\gamma_{T}^{*} \rightarrow V_{L}\right)+\varepsilon d \sigma\left(\gamma_{L}^{*} \rightarrow V_{L}\right)}{d \sigma}
$$

for the $\omega$ production are shown in Fig. 7, (right). The $r_{00}^{04}$ SDME is connected with the $R$ ratio and we find similar results for both observables, see Fig 7.

The SDME $r_{1-1}^{1}$ shows the difference of the natural and unnatural parity contributions

$$
r_{1-1}^{1}=-\operatorname{Im} r_{1-1}^{2}=\frac{d \sigma^{N}\left(\gamma_{T}^{*} \rightarrow V_{T}\right)-\sigma^{U}\left(\gamma_{T}^{*} \rightarrow V_{T}\right)}{2 d \sigma} .
$$

Results for this SDMEs for the $\omega$ production are shown in Fig. 8, (left). We see that the PP effects are very strong here. With PP we find $r_{1-1}^{1} \sim-0.2$ and without PP $r_{1-1}^{1} \sim 0.2$. For the $\rho$ meson production results are shown on Fig. 9, (right). The PP contribution is small here and the results are close the to $\omega$ case without PP. 

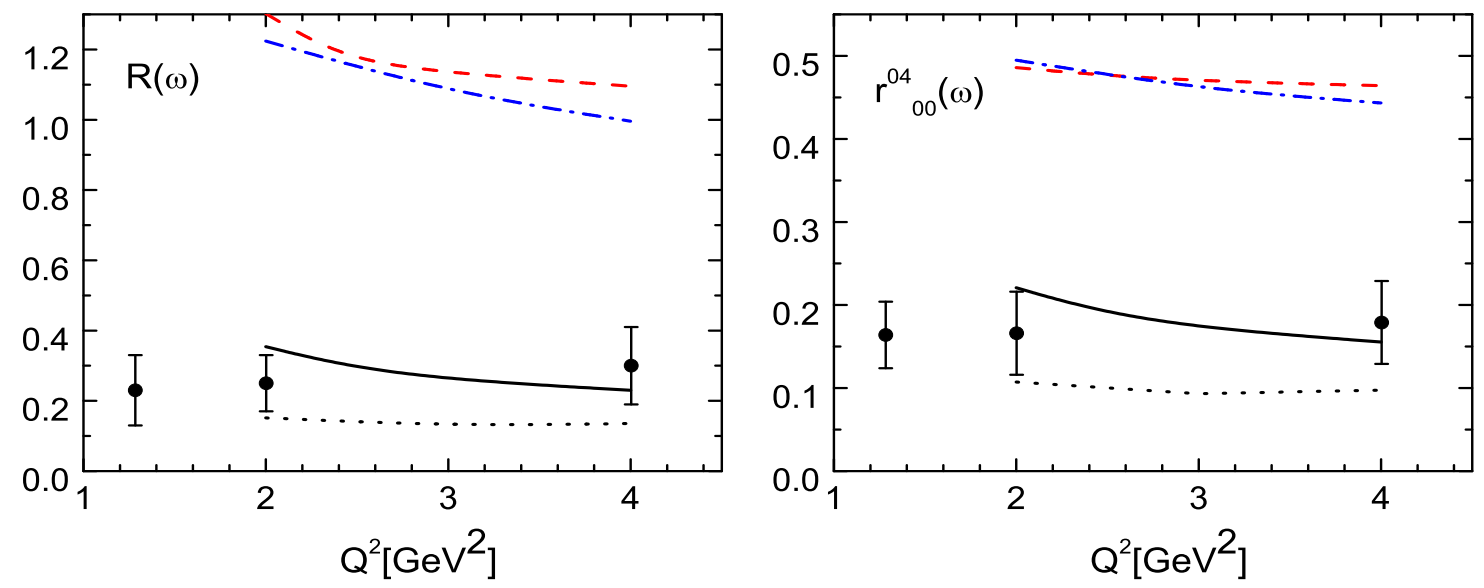

Figure 7: Left: $R(\omega)$; Right: $r_{00}^{04}(\omega)$ at HERMES: Black solid with PP, Red-dashed -without PP. Black dotted-for $W=3.5 \mathrm{GeV}$ (CLAS), Blue dashed-dotted for $W=8 \mathrm{GeV}$ (COMPASS).
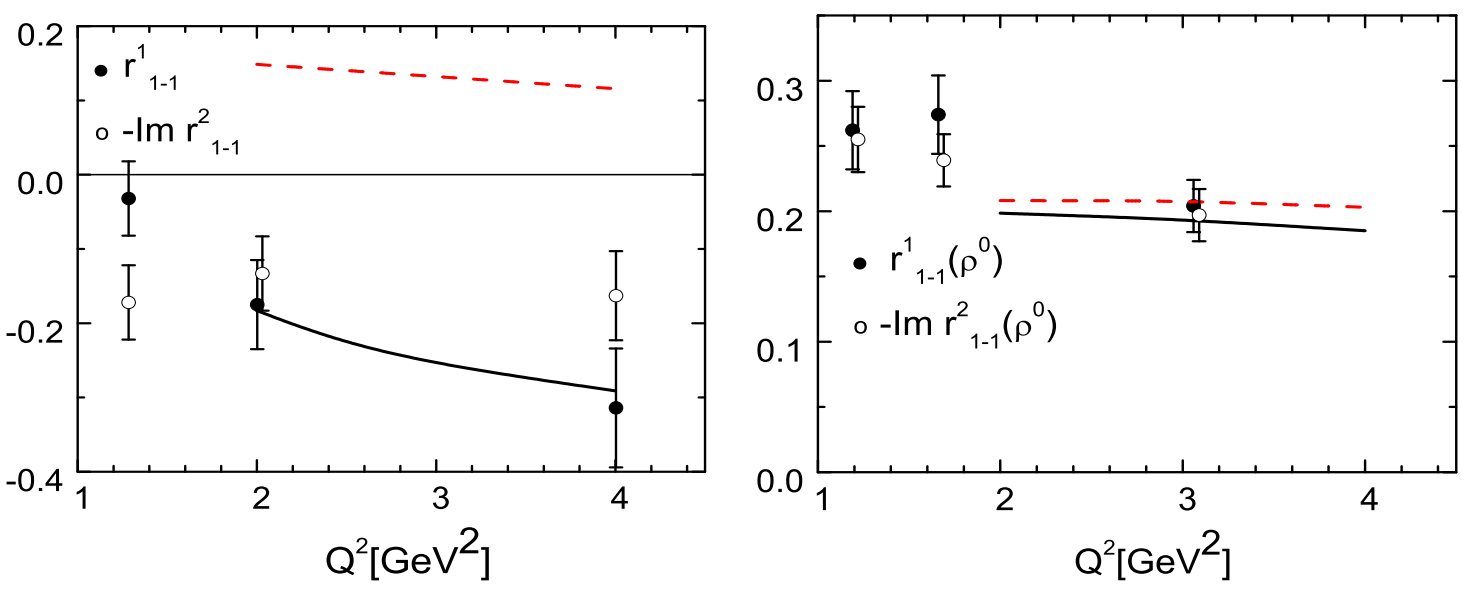

Figure 8: Left: SDMEs for $\omega$ at HERMES: Right: SDMEs for $\rho$. At HERMES: Black solid with PP, Red-dashed -without PP.

The SDME Imr $r_{1-1}^{6}$ is proportional to interference of two UP amplitudes

$$
\operatorname{Im} r_{1-1}^{6} \sim \operatorname{Re} M^{U}(+-++) M^{U}(+-0+) .
$$

Our model results show that the PP contribution to unnatural parity amplitudes describes well the $Q^{2}$ and $t$ dependences of $\operatorname{Im} r_{1-1}^{6}$ SDME, see Fig. 9. Without PP this SDME is equal to zero.

\section{Conclusion}

The exclusive electroproduction of vector and pseudoscalar mesons was analyzed here within the handbag approach where the amplitude factorizes [2] into the subprocess amplitudes and GPDs, which contain information about the hadron structure. The hard subprocess amplitude is calculated within the $k_{\perp}$ factorization scheme [3]. The results based on this approach on the VM cross sections 

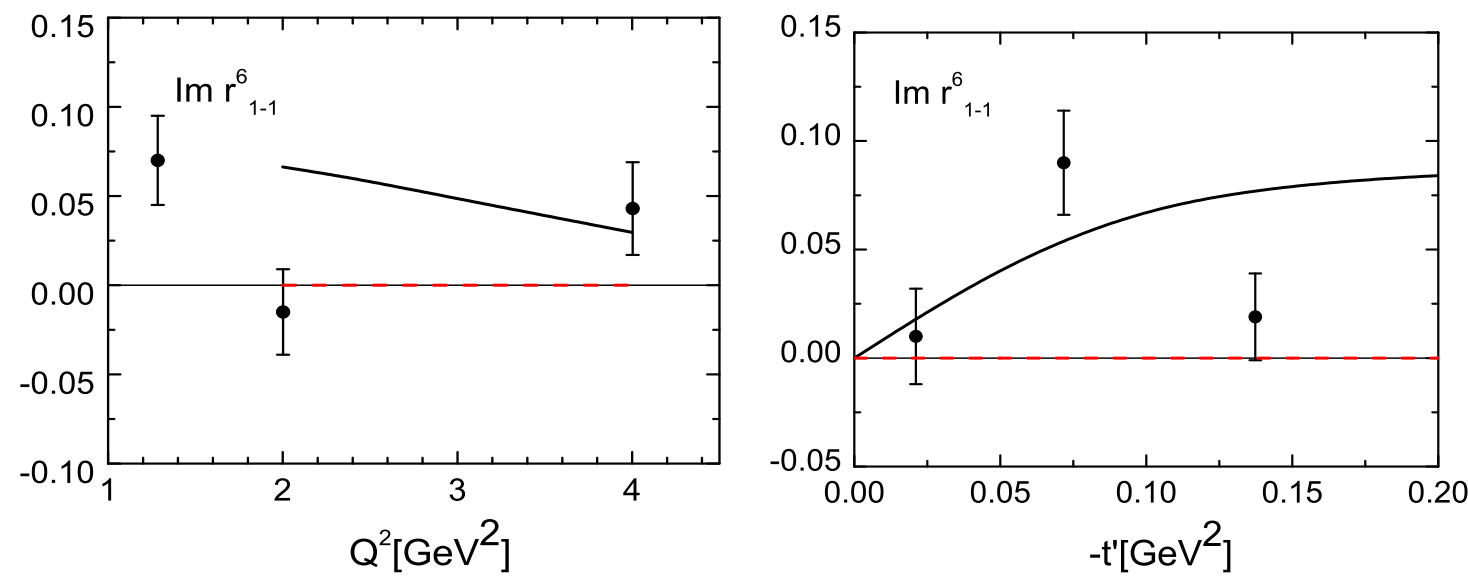

Figure 9: SDME Im $r_{1-1}^{6}$ for $\omega$ at HERMES: Left: $Q^{2}$ dependence of SDME ; Right: $-t$ dependence of SDMEs: Black solid with PP, Red-dashed -without PP

and various spin observables are in good agreement with data at HERMES, COMPASS and HERA energies at high $Q^{2}[1]$.

The role of transversity $H_{T}$ and $\bar{E}_{T}$ GPDs in leptoproduction of light mesons was investigated within the framework of the handbag approach in $[4,5]$. The transversity GPDs in combination with twist- 3 meson wave functions occur in the amplitudes for transitions from a transversely polarized virtual photon to a longitudinal polarized vector meson. It was found that the transversity effects are essential in the PM leptoproduction where they lead to large transverse cross sections, which exceed substantially the leading twist longitudinal cross section. There is an experimental indication that the transversity effects in the PM production were likely observed in CLAS [14]. In the VM production, transversity contributions were analysed [16] in SDMEs and in asymmetries measured with a transversely polarized target where such effects are essential. The results are consistent with HERMES and COMPASS data $[17,18]$ on the $\rho^{0}$ production.

Using GPDs from our analyses of hard meson leptoproduction we investigated the $\omega$ SDMEs [6] measured by the HERMES Collaboration [7]. It was found that PP give an essential contributions to the $\omega$ production. The PP contribution explains the large unnatural-parity effects which exceed the natural parity contribution in the $\omega$ production, as observed by HERMES. For example the ratio of the unnatural to the natural parity cross section was found for $\omega$ to be larger than unity instead of the expected small value. Results for the $\rho$ production were presented too. The PP contribution in the $\rho^{0}$ production is much smaller with respect to the $\omega$ case, which is consistent with experiment [17]. Our results [6] are in good agreement with the HERMES experimental data $[7,17]$.

This work is supported in part by the Russian Foundation for Basic Research, Grant 12-0200613 and by the Heisenberg-Landau program. 


\section{References}

[1] S.V. Goloskokov, P. Kroll, Euro. Phys. J. C42 (2005) 281;

S.V. Goloskokov, P. Kroll, Euro. Phys. J. C50 (2007) 829;

S.V. Goloskokov, P. Kroll, Euro. Phys. J. C53 (2008) 367;

S.V. Goloskokov, P. Kroll, Euro. Phys. J. C59 (2009) 809.

[2] X. Ji, Phys. Rev. D55 (1997) 7114;

A.V. Radyushkin, Phys. Lett. $\mathbf{B 3 8 0}$ (1996) 417;

J.C. Collins, et al., Phys. Rev. D56 (1997) 2982.

[3] J. Botts and G. Sterman, Nucl. Phys. B325 (1989) 62.

[4] S.V.Goloskokov, P.Kroll, Euro. Phys. J. C65 (2010) 137.

[5] S.V.Goloskokov, P.Kroll, Euro. Phys. J. A47 (2011) 112.

[6] S.V Goloskokov, P. Kroll, Euro. Phys. J. A50 (2014) 146.

[7] A. Airapetian et al. (HERMES Collab.), Eur.Phys.J. C74 (2014) 3110

[8] J. Bolz, J.G. Körner and P. Kroll, Z. Phys. A350 (1994) 145.

[9] I.V. Musatov and A.V. Radyushkin, Phys. Rev. D61 (2000) 074027.

[10] J. Pumplin, et al., JHEP 0207 (2002) 012.

[11] M. Diehl, T. Feldmann, R. Jakob and P. Kroll, Euro. Phys. J. C39 (2005) 1; M. Diehl, P. Kroll, Eur.Phys.J. C73 (2013) 2397.

[12] M. Anselmino, M. Boglione, U. D’Alesio, A. Kotzinian, F. Murgia, A. Prokudin and S. Melis, Nucl. Phys. Proc. Suppl. 191 (2009) 98.

[13] M. Gockeler et al. (QCDSF Collaboration and UKQCD Collaboration, Phys. Rev. Lett. 98 (2007) 222001.

[14] I. Bedlinskiy, et al. (CLAS Collaboration) Phys. Rev. Lett. 109 (2012) 112001.

[15] V.Kubarovsky et al., Proc. of DSPIN-11, Dubna, September 2011, p. 258.

[16] S.V Goloskokov, P. Kroll, Euro. Phys. J. C74 2725 (2014).

[17] A. Airapetian et al. (HERMES Collab.), Euro. Phys. J. C62 (2009) 659.

[18] C. Adolph, et al. (COMPASS Collaboration), Phys.Lett. B731 (2014) 19. 